\title{
Gold Nanoparticles Incorporated with Cyclodextrins and Its Applications
}

\author{
Fahad Abdulaziz1, Dina Salah ${ }^{2 *}$ \\ ${ }^{1}$ Department of Chemistry, College of Science, University of Hail, Hail, Saudi Arabia \\ ${ }^{2}$ Biophysics Group, Physics Department, Ain Shams University, Cairo, Egypt \\ Email: fah.alanazi@uoh.edu.sa, *dinasalah@sci.asu.edu.eg
}

How to cite this paper: Abdulaziz, F. and Salah, D. (2021) Gold Nanoparticles Incorporated with Cyclodextrins and Its Applications. Journal of Biomaterials and Nanobiotechnology, 12, 79-97. https://doi.org/10.4236/jbnb.2021.124007

Received: September 27, 2021

Accepted: October 26, 2021

Published: October 29, 2021

Copyright $\odot 2021$ by author(s) and Scientific Research Publishing Inc. This work is licensed under the Creative Commons Attribution International License (CC BY 4.0).

http://creativecommons.org/licenses/by/4.0/

(c) (i) Open Access

\begin{abstract}
Cyclodextrins are naturally oligosaccharides which had cyclic glucopyranoside units even if six units to form $\alpha$-cyclodextrin $(\alpha$-CD), seven units $\beta$-cyclodextrin $(\beta$-CD) or eight units $\gamma$-cyclodextrin $(\gamma$-CD) and some other derivatives. It has a truncated cone shape with a hydrophobic cavity and a hydrophilic from the external surface of cyclodextrins. Gold nanoparticles incorporated with cyclodextrins enhanced optical and electrical properties of the resulting conjugates, due to the gold nanoparticles' unique physical and chemical properties because of their surface plasmon resonance. Here is a review of gold nanoparticles/cyclodextrin's different applications including sensing, antimicrobial effect, and their variable medical/pharmaceutical applications. Generally, gold nanoparticles and cyclodextrin conjugates showed developed and pronounced advantages due to their biocompatibility and enhanced physical and electric properties.
\end{abstract}

\section{Keywords}

Gold Nanoparticles, Cyclodextrins, Sensors, Antimicrobe, Biomedicine

\section{Introduction}

\subsection{Cyclodextrins}

Cyclodextrins (CDs) are cyclic oligosaccharides which naturally obtained from materials that contain starch by an enzymatic reaction to form at least six glucose units to form $\alpha$-cyclodextrin $(\alpha-\mathrm{CD})$, seven glucose units $\beta$-cyclodextrin $(\beta$-CD) or eight glucose units $\gamma$-cyclodextrin $(\gamma$-CD), cyclodextrins are water-soluble cyclic oligosaccharides with a truncated cone shape, tapered hydrophobic cavity length of $8.0 \AA$ and two rims of hydroxyl groups [1].

The external surface of cyclodextrins is hydrophilic that offers protons (H1, 
$\mathrm{H} 2, \mathrm{H} 4, \mathrm{H} 6$ ), whereas the inner cavity is hydrophobic due to the presence of rings of $\mathrm{C}-\mathrm{H}$ groups, and it offers ( $\mathrm{H} 3$ and $\mathrm{H} 5$ ) [2]. The top and bottom diameter of these cyclodextrins are 4.7 and $5.3 \AA$ for $\alpha$-CD, 6.0 and $6.5 \AA$ for $\beta$-CD, and 7.5 and $8.3 \AA$ for $\gamma$-CD respectively [3]. Therefore, each of these cyclodextrins or their derivatives have their own ability to include guests of different polarity and dimensions, depending on the size compatibility of the guest and the hydrophobic cavity of the host. Figure 1 shows a schematic diagram for different cyclodextrins (CDs).

Cyclodextrins, calixarenes, cucurbit[n]urils, dendrimers, and other host systems have been used to accommodate guest molecules. However, cyclodextrins have been shown to be the most popular macrocyclic host material. Coelho and coworkers discussed encapsulation of guest's molecules into host materials as a major photo stabilization method for photosensitive materials. They reported that cyclodextrins are the most used to encapsulate different guest molecules. The great structural features of these materials enhance the catalytic and photophysical properties allowing them to be used in many pharmaceutical and cosmetic products. Various guest molecules have been encapsulated into different host molecules previously but with less frequency than cyclodextrins such as liposomes, lipid nanoparticles, polymeric nanoparticles, and mesoporous silica nanoparticles [4].

\subsection{Cyclodextrins and Its Applications}

Das et al. studied the inclusion of insoluble drug (ibuprofen) in captisol (sulfobutylether sodium salt of $\beta$-CD), the stability of this complex increases at double distilled water if compared to phosphate buffer which its $\mathrm{pH} 7.2$, at double distilled water ibuprofen is on the non-ionized form. They used two different techniques to prepare the drug complex, and their results show improvement of drug
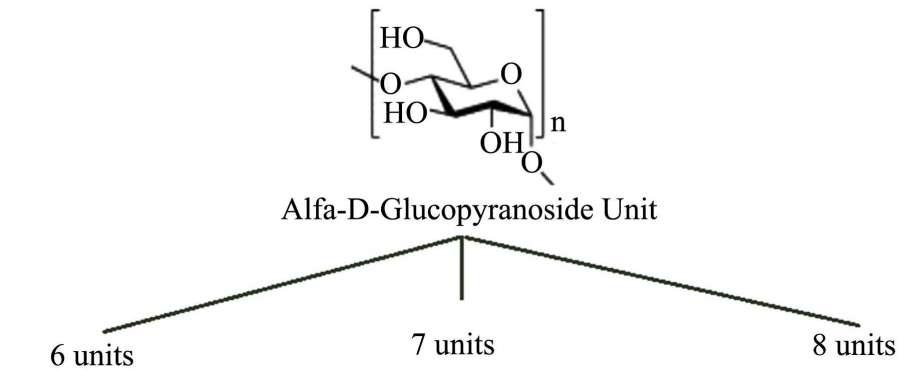

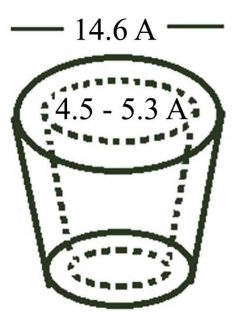

Alfa Cyclodextrin

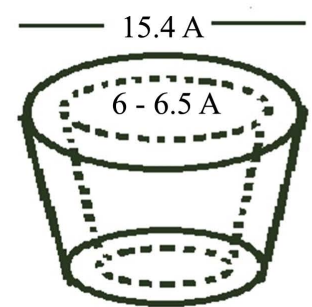

Beta Cyclodextrin

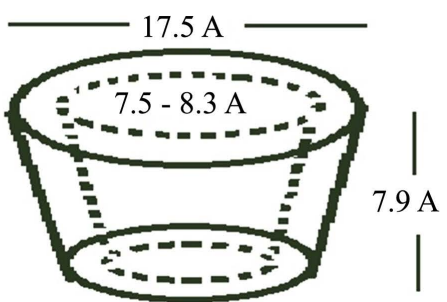

Gamma Cyclodextrin

Figure 1. A schematic diagram for different cyclodextrins. 
dissolution for the samples prepared by freeze-drying if compared to the kneading technique. The drug- $\beta$ cyclodextrin complex forms 1:1 stoichiometric inclusion. Their results are based on Fourier transform infrared spectroscopy (FT-IR), Differential scanning calorimetry (DSC), X-ray powder diffractometry (pXRD), Scanning electron microscope (SEM), and Molecular docking studies [5].

Adeoyea and coworkers used the supercritical $\mathrm{CO}_{2}$-assisted spray-drying (SASD) technique to form a complex by inclusion of ibuprofen into hydroxypropyl- $\gamma$-cyclodextrin (HP- $\gamma$-CD), they used different methods to support their results such as infrared spectroscopy (ATR-FTIR), X-ray diffraction (XRD), ultraviolet spectroscopy (UV), cross-polarization magic angle spinning nuclear magnetic resonance (CP/MAS NMR), differential scanning calorimetry (DSC) and morphological studies. Their results show a good, amorphous, with homogenous particle size and a clean formation of drug/polymer complex. They show how a SASD technique can be a promising alternative for conventional spray drying and other methods of CDs solid complex formation, as it is non-toxic, more convenient for thermolabile preparation. The most important perspective results for this technique are homogenous and controlled particle size, which is with a great advantage to enhance pulmonary and nanoparticles drug delivery complexes [6].

2R, 3R-Dihydromyricetin (DMY) is a natural flavonoid that has different biological activities, this DMY has low chemical stability and low water solubility, these characteristics limit their applications in food and pharmaceutical fields. $\mathrm{Wu}$ et al., show improved (DMY) solubility, stability, antioxidant, and lipid-lowering activities upon its inclusion using different cyclodextrins ( $\alpha-\beta$ and $\Upsilon \mathrm{CDs}$ ) and their derivatives, these results are based on FTIR, PXRD, DSC, SEM, and NMR analysis [7].

Guzzo and coworkers introduce a detailed study for the conformational changes for the inclusion of Ketoprofen (Kp) in $\beta$-Cyclodextrin following it using NMR spectroscopy and its coloration with molecular dynamic simulations. Following upfield and downfield shifts show a slight reduction in the angle between the two phenyl rings of ketoprofen and a significant reduction in the mobility of the carboxyl group. The $\mathrm{Kp} / \beta \mathrm{CD}$ complex shows improvement in its photodecarboxylation upon UV exposure and less degradation [8].

Also, Yang et al. studied the inclusion of mangiferin which has its role as a hypoglycemic agent, an antioxidant, and an anti-inflammatory agent. Mangiferin inclusion in $\beta$-cyclodextrin and its derivatives such as hydroxypropyl- $\beta$ cyclodextrin (HP $\beta C D$ ), Sulfobutyl ether $\beta$-cyclodextrin $(\mathrm{SBE} \beta \mathrm{CD})$ and mono (6-ethylene-dismino-6-deoxy)- $\beta$-cyclodextrin (EN $\beta C D)$. Their results show good stability and solubility of the complexes in water [9].

Pereva et al. studied Ibuprofen inclusion in $\beta$ cyclodextrin by a combination of theoretical and experimental NMR (2D-ROESY) studies. The theoretical calculations show that the less polar part of the ibuprofen is inserted into the cyclodextrin cavity, their results also show stability of the formed complex even if 
the polarity of the solvents used is changed [10].

Capsaicin is used as a temporary relief of muscle or joint pain caused by strains. Capsaicin topical cab is also used to treat nerve pain Ciulu-Costinescu et al., improve its solubility by its inclusion by 1:1 molar ratio with $\alpha$ cyclodextrin and study its effect as anti-microbial associated infection resulted from four different gram-positive and negative bacteria [11].

Naringenin is a flavonoid compound present in different citrus fruits, although it has anti-inflammatory, antioxidant effects its clinical uses are limited due to its poor solubility and stability. Guan et al., study improvement of its clinical application for pulmonary delivery by its inclusion into $\mathrm{Hp} \beta C D$. They used different techniques in their studies such as differential scanning calorimetry (DSC), proton nuclear magnetic resonance (1HNMR), XRD, and two-dimensional rotating frame Overhauser effect spectroscopy (2D ROESY) which confirm 1:1 stoichiometry and complete inclusion of naringenin into a HBCD cavity. To show the cellular uptake of the inclusion complex in-vitro study shows concentrated of naringenin/HpBCD in the epithelium-lining fluid (ELF) of Calu-3 cells. Sprague-Dawley (SD) rats were used for the pharmacokinetic in-vivo study [12].

Ivansyah et al. used the density functional theory computational method to study the inclusion of $\mathrm{R}$ and $\mathrm{S}$ omeprazole in $\beta$-Cyclodextrin, omeprazole used as a medication for gastroesophageal reflux disease, peptic ulcer disease, and Zollinger-Ellison syndrome. It is also used to prevent upper gastrointestinal bleeding in certain patients. Their results show that the complexes formed by van der Waals interaction and steric effect can be ignored as their amount is relatively small [13].

Carneiro et al. published a review discussing the role of cyclodextrin to enhance the chemical and biological characteristics of inclusion complexes as well as increase their solubility and stability in aqueous solutions. These inclusion complexes show a good bioavailability which makes cyclodextrins a very promising polymer that can be used in pharmaceutical area [14].

Gentili reported a promising different application of cyclodextrin as it is low price oligosaccharide, environmentally biocompatible, and naturally degradation compound. There are different approaches to use cyclodextrins first immobilization onto inactive materials such as silica; second, immobilization onto nanomaterials such as graphene oxide and different nanoparticles; the third polymerization with specific cross-linkers to form the so-called CD-based nanosponges. This can be applied in different fields [15].

García-Pérez studied the use of elicited plant suspension-cultured cells (PSCC) with cyclodextrins (CDs) as an alternative method to produce bioactive compounds such as polyphenols, cyclodextrins shift the homeostatic equilibrium of the cells which eventually stressed the cells to produce more phenolics and flavonoids compounds, as well as increased of scavenging activity of these active compounds due to host-guest inclusion into cyclodextrin [16].

Paczkowska et al. Studied the tebipenem pivoxil (orally administrated antibi- 
otic) enhancement of its physical, chemical, and biological properties upon its inclusion into $\beta$-CD. Forming host/guest complex physicochemical properties enhanced tebipenem pivoxil solubility and thermal stability, as well as increased its antibacterial activity and its prolonged release into the intestinal environment [17].

de Almeida Magalhães and coworkers studied the inclusion of nonpolar Euterpe oleracea Mart oil (EOO) into $\beta$-cyclodextrin and its derivatives. Their studies are based on theoretical molecular modeling. Fourier transforms infrared spectroscopy, X-ray diffraction, thermogravimetry analysis, differential scanning calorimetry, and scanning electron microscopy. They also study its antibacterial activity against four different bacterial strains Escherichia coli, Streptomyces aureus, Pseudomonas aeruginosa, and Enterococcus faecalis which shows enhance of the antimicrobial response of the inclusion complex against these bacterial strains. This inclusion introduced and developed new drugs and pharmaceutical forms towards its application in medicine [18].

da Cunha Trajano reported the slow delivery system of doxycycline (DOX) inclusion in $\beta$-cyclodextrin $(\mathrm{DOX}) / \beta \mathrm{CD}$ ) compared to $\mathrm{DOX}$ alone in a gel. These results show a promising application for the Dox inclusion in $\beta \mathrm{CD}$ in the dentistry field as this complex enhances and increases bone density due to DOX anti-inflammatory effect [19].

Santos et al. studied the inclusion of xanthine into HP- $\beta$-CD which has a large cavity volume. Xanthine is a group of alkaloids commonly used as a mild stimulant and as a bronchodilator, particularly in the treatment of asthma or influenza symptoms. Their results are based on the analysis of the molecular mechanisms for the complex formation by examining their density, molar volume, viscosity, temperature, and diffusion coefficient [20].

Cordycepin is a very important medicinal compound used for antitumor, which has been found to exert anti-angiogenic, anti-metastatic, and antiproliferative effects, as well as inducing apoptosis. Zhang et al. study its inclusion into cyclodextrins as its purine group is deeply inserted into the CDs cavity and form 1:1 stoichiometry. These results were obtained by using different spectroscopic techniques such as UV-Vis, FTIR and HNMR, in addition to DSC, SEM, XRD, ESI-MS techniques [21].

Ren et al. increased the water solubility and light stability of rubropunctain as an anticancer drug upon its inclusion in water-soluble $\beta$-cyclodextrin. These results are based on FESEM, FTIR and XRD. Their results also show an increase of rubrropuncatin cell viability when tested on Hela cells as the use of an organic solvent such as DMSO to dissolve this chemotherapy drug was eliminated [22].

Ko and coworkers introduce a new antitumor active complex to treat HER2positive breast cancer. This hos/guest complex delivers both Herceptin and doxorubicin into cancer cells upon conjugation with $\beta$-CD and carbon-based nanoparticles. A high concentration of antitumor drugs was released into the cancer cells were triggered due to glutathione's high concentration and low $\mathrm{pH}$ level at cancer cells. These results were confirmed by cell viability tests, confocal laser 
scanning microscopy, and in-vivo animal studies [23].

Bezamat et al. studied the inclusion of pramoxine which is a local anesthetic agent used in clinical operations to eradicate pain. The use of pramoxine is limited as its chemical structure has a morpholine ring which makes it less soluble in water as well as its invitro toxicity. Inclusion of pramoxine at hydroxypropyl- $\beta$-cyclodextrin (HP- $\beta$-CD) shows $1: 1$ molar ratio, as its aromatic ring is embedded into the $\mathrm{Hp}-\beta$-CD cavity. These results were confirmed using different methods such as X-ray diffractogram, $1 \mathrm{H}$ NMR (DOSY), Also bioavailability increases upon inclusion if compared to pure pramoxine, as it returned human erythrocyte hemolysis [24].

Moradi et al. studied the inclusion of Thyme oil into different cyclodextrins and its derivatives as methyl- $\beta$-, hydroxypropyl- $\beta$ - and $\gamma$-cyclodextrin. These inclusion compounds were incorporated into $\mathrm{pH}$-sensitive chitosan/polyvinyl alcohol hydrogel with good mechanical and swelling characteristics. This hydrogel/inclusion complexes system can be used as wound dressing which has a controlled release of the drug and shows good antibacterial activity, specifically the inclusion in $\gamma$-CD if compared to $\beta$-CD as regarded to antibacterial activity [25].

Andriotis et al. reported cyclodextrin/chitosan/propolis complexes used as antimicrobial wound dressing film. These patches show good cytocompatibility and antimicrobial, also enhanced skin surface hydrophobicity, opacity and mechanical characteristics [26].

Fenyvesi and coworkers published a review article discussing the cyclodextrins application in the food industry. Cyclodextrins are test-less, order-less, non-caloric and non-cariogenic saccharides which non or partially digested by gastrointestinal tract enzymes and fermented by the gut micoflora, so they can be used as dietary fibers for body weight and blood lipid profile control. So, these cyclodextrins are anti-obesity and anti-diabetic compounds, their mechanism of action as bioactive food supplements based on; first their action as solubilizers and stabilizer for dietary lipids such as unsaturated fatty acids, vitamins, flavonoids, carotenoids, and phytosterols; second is its reduction for trans-fats allergens, acrylamides, and mycotoxins [27].

Pourreza et al. used D-limonene and $\beta$-carotene to determine the concentration of $\beta$-cyclodextrin using dispersive liquid to liquid microextraction spectroscopic method. They tested different $\mathrm{PH}$, nature, and volume of the sample solution and report $4 \times 10^{-5} \mathrm{~mol} \cdot \mathrm{L}^{-1}$ as a detection limit for $\beta$ - $\mathrm{CD}$ in solution [28].

Siva et al. introduced ultrasonic as high efficiency and environment friendly, and less energy consuming method for preparation of some low solubility essential and applicable oils derived from medicinal plants such as cuminaldehye and isoeugenol. The antibacterial effect of the prepared complexes was tested against gram-positive and gram-negative bacteria and showed its bioavailability.

Using different oils as an alternative natural drug and it's used in different food, pharmaceutical, and cosmetic industries, these oils suffer from their low solubility in aqueous solution, low stability, and evaporation. da Silva Júnior et 
al. present a review for cyclodextrin inclusion in these oils to overcome its application limitation in different fields. The inclusion of these natural oils enhances its solubility in aqueous solution and minimizes its photodegradation which eventually increases their stability. These oils/CDs complexes show very promising antibacterial and antioxidant effects which may use in textiles and nanofibers industries [29].

Banerjee and coworkers studied both ground state and excited state energy transfer from C6- $\beta$-cyclodextrin complexes (C6- $\beta$-CD) to graphene oxide (GO) which is a good electron receptor. This $\mathrm{C} 6-\beta-\mathrm{CD} / \mathrm{GO}$ can be used as an energy collecting antenna, by using cyclic voltammetry to confirm ground state energy transfer, and excited-state energy transfer results by using steady-state and time-resolved fluorescence spectroscopy [30].

\section{Gold Nanoparticles}

The study of metallic nanoparticles started decades ago because of their unique optical properties. The photophysical properties of metallic nanoparticles result from the fact that when electromagnetic radiation excites the electron of particles, it induces coherent, resonant oscillations of the free electrons in the nanoparticles. These oscillations are known as surface plasmon resonance. This is quantitatively described by Mie theory [31].

The surface plasmon resonance of noble metals such as gold nanoparticles (GNPs) influences their unique properties, such as electric field enhancements, nano-antenna characteristics, massive light scattering/absorption, and photothermal or photochemical conversion capabilities. Among the metallic nanoparticles, gold nanoparticles are very important because of their biocompatibility which is useful in biomedical applications [32].

The plasmon surface plasmon resonance depends on the nanoparticles' geometries, as shown in Figure 2. For example, spherical gold nanoparticles have one surface plasmon around $520 \mathrm{~nm}$ which depends mainly on their diameter. Changing the spherical nanoparticles diameter from 10 to $100 \mathrm{~nm}$ changes the plasmon surface plasmon resonance position by only $50 \mathrm{~nm}$ [33], also Link and El-Sayed studied the relation between the bandwidth and the size of nanoparticles and revealed that an inverse relationship has been observed when the nanoparticles are less than $20 \mathrm{~nm}$ in diameter. Interestingly, a proportional relationship also was observed when the size of nanoparticles is larger than $20 \mathrm{~nm}$ [34]. The results confirm Mie theory regarding to the linear dependence of absorption coefficient with the volume of the nanoparticles [34]. Gold nanoparticles can be synthesized in different shapes nanorods, cubes, stars, etc. Gold nanorods have anisotropic plasmon resonances related to electron oscillation along with two different directions, the transverse surface plasmon resonance (TSPR) around $520 \mathrm{~nm}$ due to electron oscillation along with the short axis and the longitudinal surface plasmon resonance (LSPR) which can be tuned from 600 to $1100 \mathrm{~nm}$ due to the electron oscillation along with the long axis. 


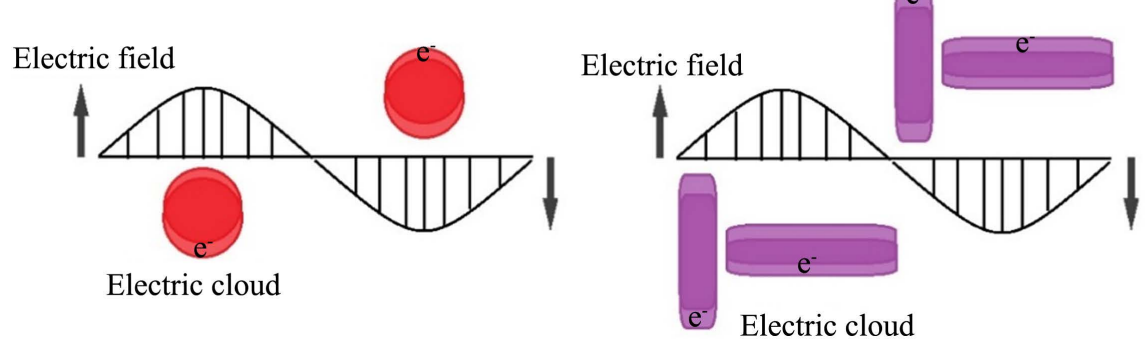

Figure 2. Surface plasmon resonance oscillations in spherical and rods gold nanoparticles.

The LSPRs of gold nanorods make them superlative candidates for biomedical imaging and therapeutic applications because they act as light absorbers above $600 \mathrm{~nm}$, where the need for near-infrared light which has larger penetration depth for biological applications is necessary, hence the light in the region between 650 - $900 \mathrm{~nm}$ has several centimeters penetration depth depending on the type of the tissue [35].

Few review articles have been discussed the incorporation of gold nanoparticles into cyclodextrin materials. We herein present a review article discussing the incorporation of gold nanoparticles and cyclodextrin and its applications in different fields such as sensing, antimicrobial and medical/pharmaceutical. The incorporated gold nanoparticles conjugates are very promising eco-friendly, low cost and available conjugates. The incorporation of gold nanoparticles and cyclodextrins enhances the physical and chemical properties that can be used in different applications.

\section{Gold Nanoparticles/CDs Sensing Applications}

Jiang et al. has been determined that thiolated $\beta$ cyclodextrin functionalized gold nanoparticles with reduced graphene oxide can be used for electrochemical sensing of acetaminophen and ofloxacin. The detection limits for them were 0.03 $\mu \mathrm{M}$ and $0.008 \mu \mathrm{M}(\mathrm{S} / \mathrm{N}=3)$ respectively. It also showed a wide linear detection range, high stability, and reproducibility. Acetaminophen is used for the treatment of arthralgia, neuralgia, cephalalgia, cancer pain, headache, backache, and postoperative pain. However, the overdoses of it cause liver and kidney problems. Ofloxacin has a broad antibacterial activity counter to gram-positive and gram-negative bacteria, so it can be used for the treatment of respiratory, urogenital, and gastrointestinal infections [36].

Shin et al. used a molecular imprinted system prepared from acryloyl-modified $\beta$-cyclodextrin ( $\beta$-CD), acrylamide (AAm), and $\mathrm{N}, \mathrm{N}$-methylenebisacrylamide (MBAA), and imprinted with bisphenol A (BPA) as shown in Figure 3 and Figure 4. The synthesized imprinted system has been utilized to sense bisphenol that is widely used in polycarbonate production and epoxy resin. It effects the human health endocrine system and therefore bisphenol is important to detect its amount in the environment. 


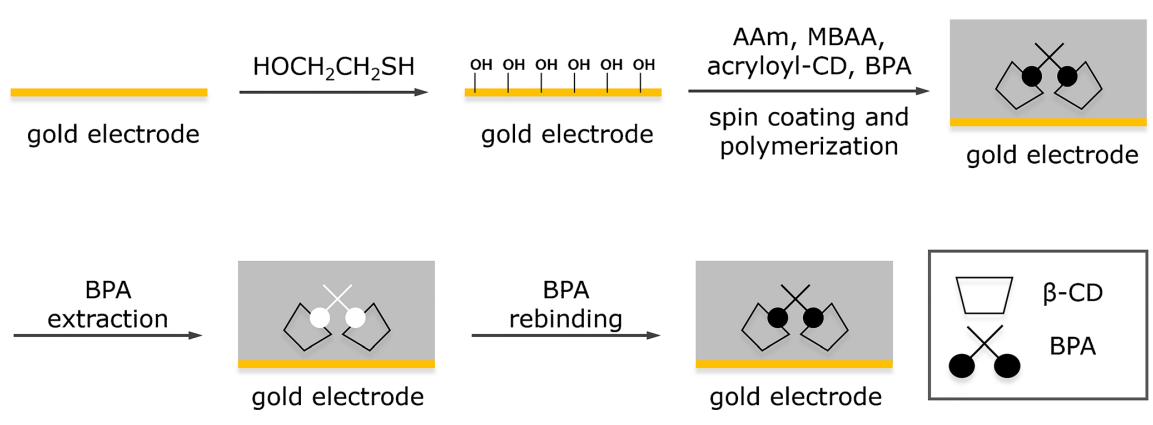

Figure 3. Fabricating a BPA-imprinted electrode using acryloyl-CD, BPA, AAm, and MBAA [37].

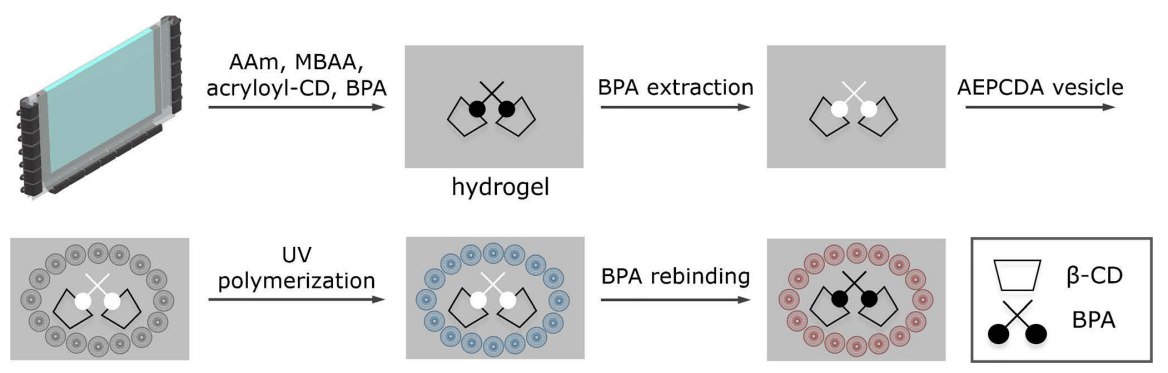

Figure 4. Fabricating a color-changeable molecular imprinting system for detecting BPA [37].

The detection of bisphenol can be achieved by two ways, first using potentiometry and then color change of the hydrogel system from blue to red, when bisphenol rebound as the membrane contracted by $35 \%$ and the color changed. The molecular imprinted system detected the bisphenol at a concentration of 0.5 $\mathrm{mM}$ with a detection limit $0.1 \mathrm{mM}$ on the visual sensor and $50 \mu \mathrm{M}$ using potentiometry [37].

Detection of bisphenol also reported by Yun and coworkers has been utilized microwave heating method followed by electrodeposition to modify glassy carbon electrode an electrochemical sensor. The sensor is based on $\beta$ cyclodextrin functionalized reduced graphene oxide gold nanoparticles nanocomposite and is used for ultrasensitive detection of bisphenol. The synthesized nanocomposite was characterized using Raman spectroscopy, UV-Vis spectroscopy, imaged using SEM, and further characterization by using XRD. Cyclic voltammetry and differential pulse voltammetry were used to study the proposed sensor. The results at different real samples show good linearity at a concentration range from 0.01 to $50 \mu \mathrm{M}$, a detection limit to $0.003 \mu \mathrm{M}$, good stability, and repeatability [38].

Luo et al. have been introduced an electrochemical sensor to detect Tetrabromobisphenol A (TBBPA), which used in buildings materials, plastics, printed circuit boards so it is widely presented in water, soil, animals, and human. The increased levels of TBBPA had a toxic effect on the liver, immune, and nervous system. The electrochemical sensor based on Cetyl Trimethyl Ammonium Bromide $(\mathrm{CTAB})$ functionalized gold nanoparticles-thio- $\beta$-cyclodextrin/graphene 
oxide modified glassy carbon electrode. Different sizes of gold nanoparticles were tested, and the optimum enhancement was detected for the smallest size of $6.2 \mathrm{~nm}$ which effectively accelerate the electron transfer through the sensor system. They also tested the effect of different $\mathrm{pHs}$ and the accumulations time on the TBBPA detection. The results have been illustrated a good agreement with the results obtained from high-performance liquid chromatography HPLC. The best $\mathrm{pH}$ condition was at $\mathrm{pH}=7$ and the accumulation time $=180 \mathrm{~s}$. the electrochemical sensor showed a low detection limit $1.2 \times 10^{-9} \mathrm{~mol} \cdot \mathrm{L}^{-1}$ with good linearity [39].

In addition, Manickam and coworkers synthesized a biocompatible hydrogel network integrated with gold nanocubes (AuCNs) as a biosensor to detect hydrogen peroxide $\left(\mathrm{H}_{2} \mathrm{O}_{2}\right)$ as shown in Figure 5. Detecting the change of Hydrogen peroxide levels is very important that it is involved in cytochrome $\mathrm{c}$ which is a redox-active hem protein present in the mitochondrial membrane, and it participates in electron transfer reactions of the mitochondria. Also, hydrogen peroxide is one of the most stable reactive oxygen species that can penetrate the cell membrane and cause excessive damage to the cell proteins. Graft co-polymerization of $\beta$-cyclodextrin and chitosan to form the hydrogel network. The presence of gold nanocubes improve the hydrogel conductivity (1.2 $\left.\mathrm{mA} \cdot \mathrm{mM}^{-1} \cdot \mathrm{cm}^{-1}\right)$, biosensor sensitivity with a low detection limit $\left(15 \times 10^{-9} \mathrm{M}\right)$ for hydrogen peroxide, and its cell biocompatibility [40].

Yadave et al. developed a chemo-visual sensor based on in suite synthesis of gold nanoparticles into cyclodextrin derived polymer (cross linked cyclodextrin) as shown in Figure 6. This polymer act as reducing agent to form small gold nanoparticles less than $15 \mathrm{~nm}$ and it also act as stabilizer for its surface. When

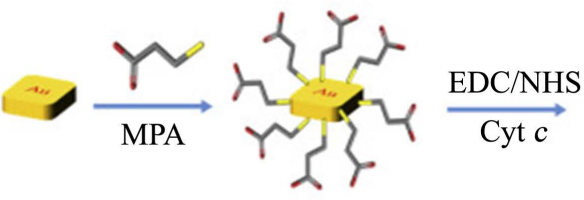

(a)

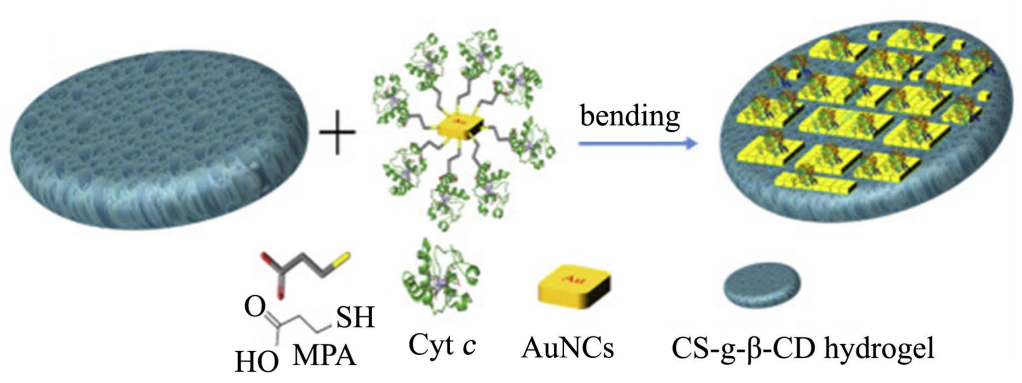

(b)

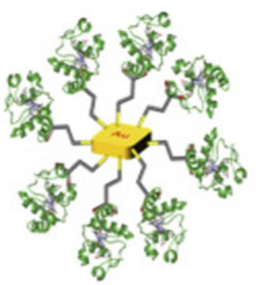

(a)

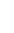




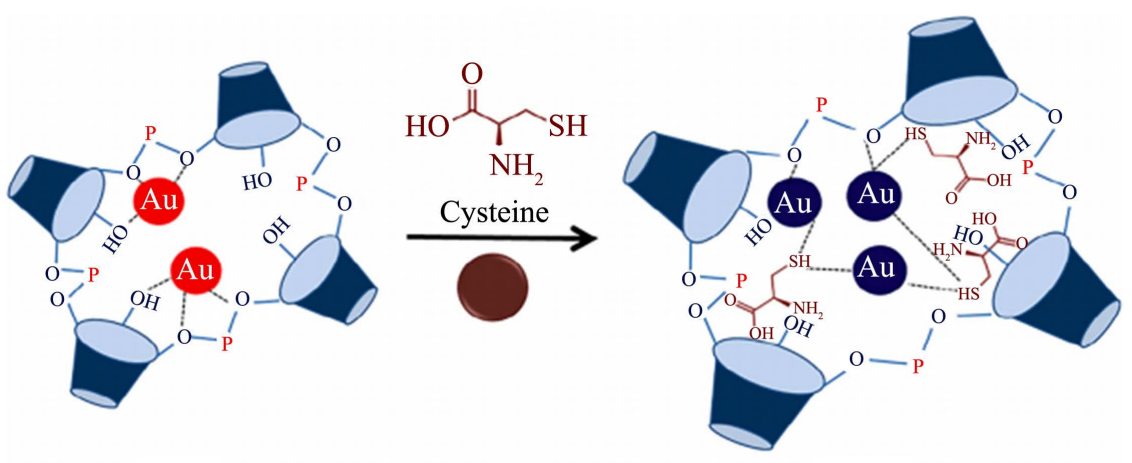

III

III
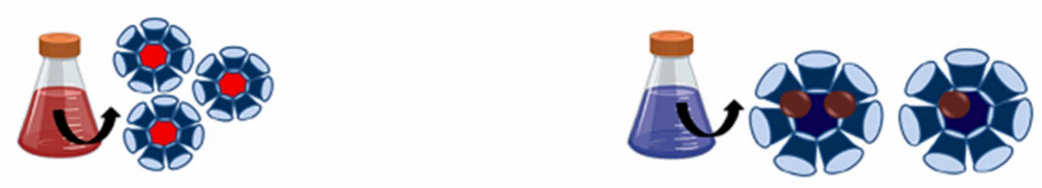

(a)

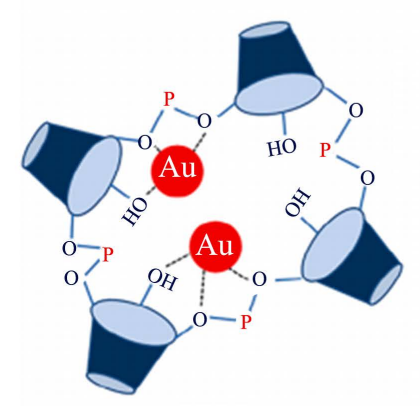

III

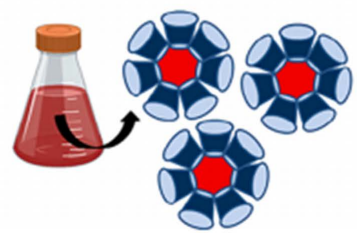

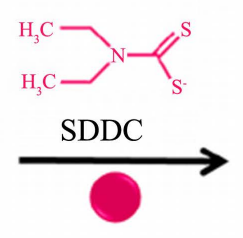

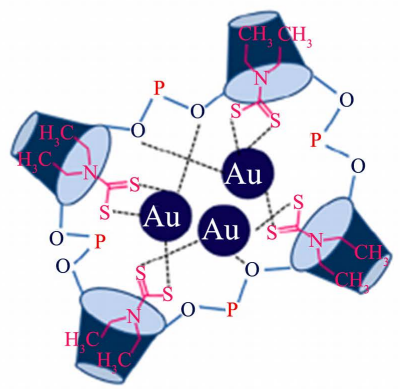

III

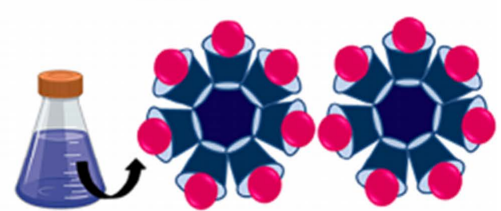

(b)

Figure 6. Schematic showing a probable mechanism for detection of (a) Cysteine and (b) SDDC using AuNS@CDPA [41].

sulfated compounds such as cysteine and SDDC (sodium diethyldithiocarbamate) attached to the gold surface due to its affinity to sulfur its color changed from red to blue. The gold nanoparticles were imaged using scanning and transmission microscopy. They suggested that the role of cyclodextrin as amphiphilic structure is to entrap the sulfated compounds and to agglomerate the gold nanoparticles. The sensor showed linearity in the range of $0.01-0.25 \mu \mathrm{M}$ for SDDC and Cysteine with detection limits values of 0.05 and $0.07 \mu \mathrm{M}$ respectively [41].

Neri et al. used cyclodextrin as reducing agent and stabilizer for the synthesis of gold nanoparticles, the direct reduction of $\mathrm{Au}^{3+}$ to $\mathrm{Au}^{0}$ to form gold nanoparticles by cyclodextrin is an alternative to chemical synthesis of gold nanopar- 
ticles. In-suit gold nanoparticles were incorporated on graphene to improve its optoelectronic properties [42].

Kapan and coworkers used drop casting method to modify the surface of graphite electrode surface. The electro-biosensor detected catechol in the linear range of $1.56 \mu \mathrm{M}-25 \mu \mathrm{M}$ catechol concentration with a limit of detection of 0.42 $\mu \mathrm{M}$ and sensitivity of $2.094 \mu \mathrm{A} \cdot \mu \mathrm{M}^{-1} \cdot \mathrm{cm}^{2}$. Catechol used in different applications and effects on the nervous system [43].

$\mathrm{Wu}$ et al. developed electrochemical biosensor to detect low-density lipoprotein (LDL) which causes atherosclerotic cardiovascular diseases. $\beta$-cyclodextrin used as receptor for lipoprotein via different binding mechanisms such as electrostatic, van der Waals forces, hydrogen bonding and hydrophobic interactions. The gold nanoparticles were deposited on stainless steel electrode to enhance the electrochemical measurements for the biosensor. The $\beta$-CD-Au modified sensor exhibited a high selectivity and sensitivity $\left(978 \mathrm{k} \Omega \cdot \mu \mathrm{M}^{-1}\right)$ toward LDL [44].

\section{Gold Nanoparticles/CDs Antimicrobial Applications}

Olmo and coworkers discussed the bacterial contaminations affecting different biomaterials used in health care even for a massive number of medical devices such as heart valves, urinary catheters, and in implantations such as dental implantations, contact, and intraocular lenses and knee and hip joints. Generally, this bacterial infection is treated by using antibiotics but the antibiotic resistance inside the biofilm still problematic. They reviewed antibacterial coating to overcome these biomaterials infections to avoid their drawbacks which eventually effect human health. Cyclodextrins were used as a system to release antimicrobial substances [45].

Raquel et al. introduced a review discussing different encapsulated systems used for food packing against microbes. Cyclodextrin and some metallic nanoparticles used as a Packing system. By encapsulation of different host materials into the CDs cavities the host optical and catalytic properties are improved, among the improver's properties of the guest molecules water solubility, protection against volatilization, oxidation, and heating. It also can be used for controlled release of host molecules out of cyclodextrin packing system. Using the cyclodextrins are with great advantages as it is low cost and biocompatible as it is a natural oligosaccharide, and it is non-toxic [46].

Bindhu and coworkers used $\beta$-cyclodextrin to synthesis and functionalize monodispersed uniform gold nanoparticles, their results were confirmed by using FTIR and UV-Vis microscopy and imaged by transmission electron microscopy, these functionalized cyclodextrin gold nanoparticles tested in many applications such as antibacterial action through inhibition of bacterial growth of E. coli and S. aureus in water, sensing of heavy metal as copper and photocatalytic degradation of textile dye in waste water [47].

\section{Gold Nanoparticles/CDs Medical Applications}

$\mathrm{Qu}$ and coworkers reported a redox responsive controlled release drug delivery 
system, mesoporous silica nanoparticles act as a vessel for the drug molecules and it was modified by ferrocene derivative groups, and gold nanoparticles functionalized by $\beta$-cyclodextrin which act as pore blockers for mesoporous silica nanoparticles, as shown in Figure 7. This blocking mechanism occurs when the ferrocene, molecules formed a stable interaction complex with $\beta$-cyclodextrin under redox conditions (uncharged ferrocene and its derivatives), while at oxidation conditions ferrocene transfers to the hydrophilic group (charged ferrocene) and therefore leave the hydrophobic cavity of $\beta$-cyclodextrin and hence loaded drug releasing. This system was tested through the addition of $\mathrm{H}_{2} \mathrm{O}_{2}$ as an oxidizing agent. The potential use of gold nanoparticles to be loaded with different drugs or molecules in addition to its functionalization with $\beta$-cyclodextrin gives the reported system a potential use in in-vivo studies [48].

Trapani and coworkers synthesized modified thiolated cyclodextrin bearing on average one thiol group at the end of an oligoethylene glycol chain per cyclodextrin unit. This modified thiolated cyclodextrin was characterized by NMR spectroscopy and MALDI analysis. The thiolated cyclodextrin was then attached to gold nanoparticles and used for controlled release of dopamine (neurotransmitter). The release of dopamine was tested using cyclic voltammetry upon stimulated the nano hybrid system by redox and digestion agents [49].

Qiu et al. introduced a safe and effective gene therapy system that depends on $\beta$ cyclodextrin modified dendrimer entrapped gold nanoparticles as shown in Figure 8. The vector system is used as cargo to deliver two therapeutic interfering RNA (siRNA), first (B-cell lymphoma/leukemia-2 (Bcl-2) siRNA and second vascular endothelial growth factor (VEGF) siRNA) to glioblastoma cells. The entrapped gold nanoparticles keep the conformation of the dendrimer, also $\beta$ cyclodextrin enhanced the cytotoxicity and the release of siRNA from endosomes after cellular uptake. Their results showed good cellular uptake of siRNA delivered by the modified gold cyclodextrin system. This modified system enhanced the gene silencing to inhibit the expression of Bcl-2 and VEGF proteins and hence can be used as a gene therapy system. Gene therapies nowadays
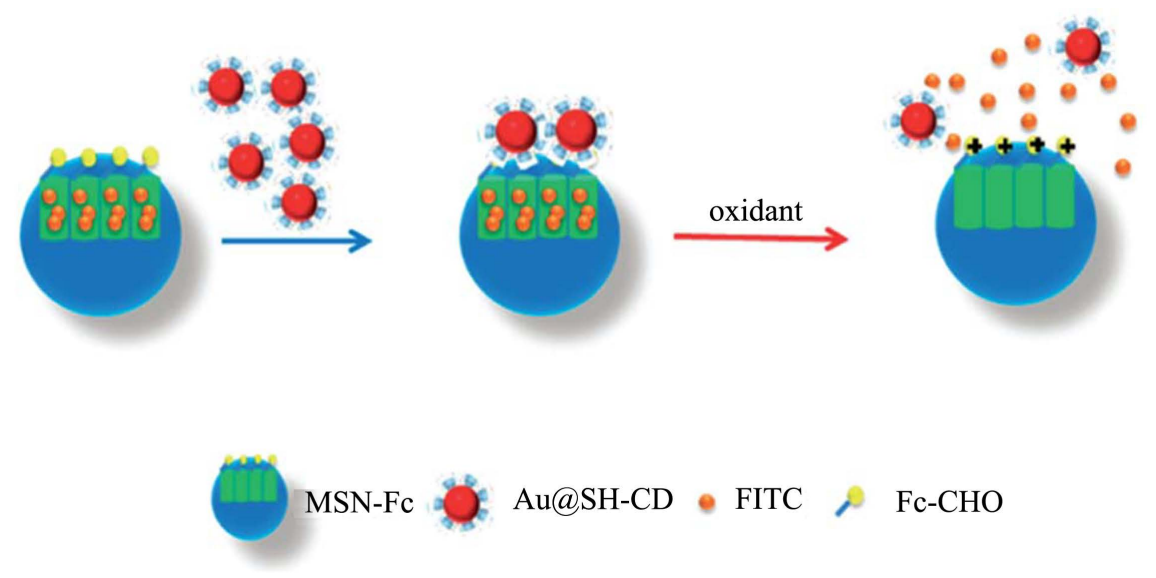

Figure 7. Schematic diagram of the redox responsive controllable release system based on the functionalized MSN and AuNP [48]. 


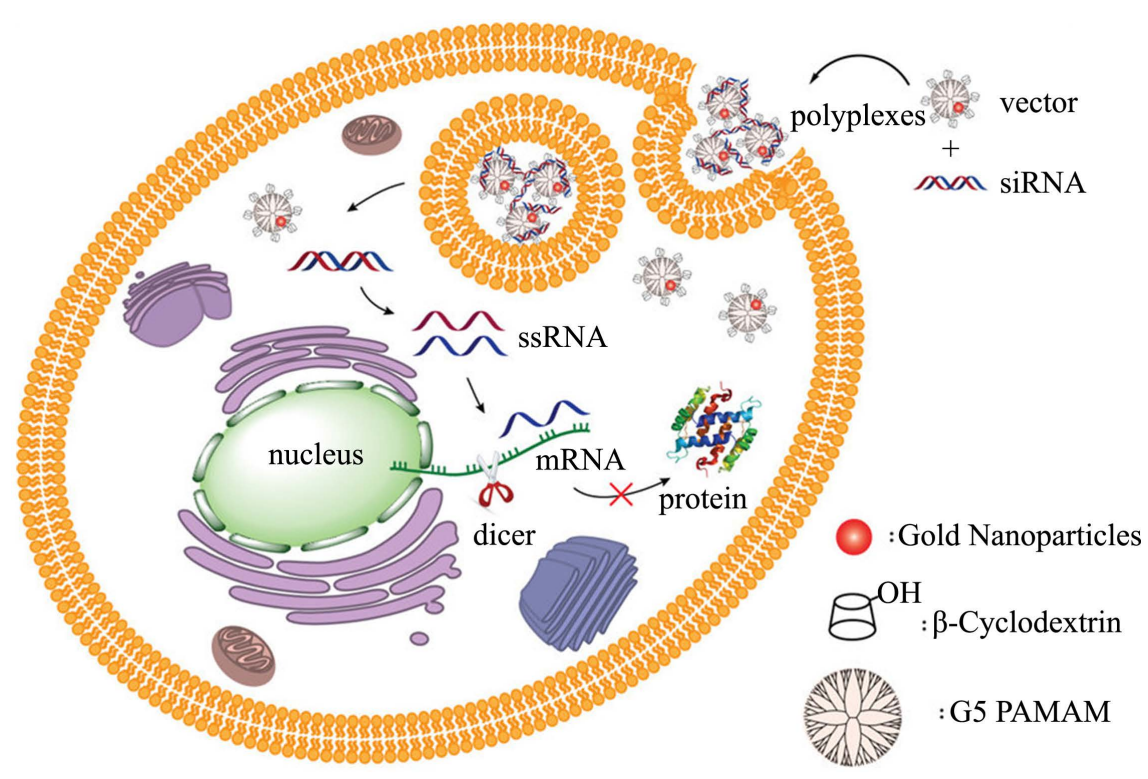

Figure 8. A Schematic illustration of the gene delivery process [50].

consider as a very promising technique for cancer therapy, using RNA interference used to silencing the specific genes which regulate cancer cells or are involved in its cell division, it is also bio-safe compared to the viral one, simple to be synthesized, and easily modified to be targeting specifically to cells. The need for a cargo or a delivery system for such promising siRNA is a must as it has a negative charge which repulsed by the negatively charged cell membrane [50].

Pestovsky and Martínez studied the controlled and long release of capsaicin by its inclusion into $\beta$ cyclodextrin. This inclusion overcomes the low solubility and gastrointestinal irritation capsaicin drawbacks which limits its use. Capsaicin is a strong spicy component in "hot" chili peppers which used for the treatment of obesity, osteoarthritis, diabetic neuropathy, post-therapy, and postherpetic neuralgia. It is also an inhibitor of platelet aggregation, induces apoptosis in cancer cells and it has an analgesic effect. They studied the capsaicin release from the inclusion complex for two days at water and acetonitrile and the presence of gold nanoparticles improve this controlled prolong release of the capsaicin and similar low molecular weight drugs [51].

\section{Conclusion}

Gold nanoparticles and cyclodextrins are very interested complexes that optimize the advantages of both. This review articles present the different applications of gold nanoparticles incorporated with cyclodextrins in such as first biosensing of different drugs such as acetaminophen ofloxacin, and sulfate-based drugs, as well as the detection of low-density lipoprotein. Second, we reported the environmental hazards detection of GNPs/CDs complexes for bisphenol, Tetrabromobisphenol $\mathrm{A}, \mathrm{H}_{2} \mathrm{O}_{2}$, catechol and heavy metals. The biological effects of GNPs/CDs complexes include forming antibacterial coatings used in food packing and in pharmaceutical applications for controlled release of different drugs 
such as dopamine, ferrocene derivatives, and capsaicin also reported. Cancer gene therapy dendrimer is considered one of the very promising medical applications for gold nanoparticles cyclodextrin dendrimer.

\section{Conflicts of Interest}

The authors declare no conflicts of interest regarding the publication of this paper.

\section{References}

[1] Connors, K.A. (1997) The Stability of Cyclodextrin Complexes in Solution. Chemical Reviews, 97, 1325-1358. https://doi.org/10.1021/cr960371r

[2] Ramos, D.S., Da Silva, P.B., Spósito, L., De Toledo, L.G., Bonifácio, B.V., Rodero, C.F., Dos Santos, K.C., Chorilli, M. and Bauab, T.M. (2018) Nanotechnology-Based Drug Delivery Systems for Control of Microbial Biofilms: A Review. International Journal of Nanomedicine, 13, 1179-1213. https://doi.org/10.2147/IJN.S146195

[3] Li, S. and Purdy, W.C. (1992) Cyclodextrins and Their Applications in Analytical Chemistry. Chemical Reviews, 92, 1457-1470. https://doi.org/10.1021/cr00014a009

[4] Coelho, L., Almeida, I.F., Sousa Lobo, J.M. and Sousa e Silva, J.P. (2018) Photostabilization Strategies of Photosensitive Drugs. International Journal of Pharmaceutics, 541, 19-25. https://doi.org/10.1016/j.ijpharm.2018.02.012

[5] Das, S.K., Kahali, N., Bose, A. and Khanam, J. (2018) Physicochemical Characterization and in Vitro Dissolution Performance of Ibuprofen-Captisol ${ }^{\circledR}$ (Sulfobutylether Sodium Salt of $\beta$-CD) Inclusion Complexes. Journal of Molecular Liquids, 261, 239-249. https://doi.org/10.1016/j.molliq.2018.04.007

[6] Adeoye, O., Costa, C., Casimiro, T., Aguiar-Ricardo, A. and Cabral-Marques, H. (2018) Preparation of Ibuprofen/Hydroxypropyl- $\gamma$-Cyclodextrin Inclusion Complexes Using Supercritical $\mathrm{CO}_{2}$-Assisted Spray Drying. The Journal of Supercritical Fluids, 133, 479-485. https://doi.org/10.1016/j.supflu.2017.11.009

[7] Wu, Y., Xiao, Y., Yue, Y., Zhong, K., Zhao, Y. and Gao, H. (2020) A Deep Insight into Mechanism for Inclusion of 2R, 3R-Dihydromyricetin with Cyclodextrins and the Effect of Complexation on Antioxidant and Lipid-Lowering Activities. Food Hydrocolloids, 103, Article ID: 105718. https://doi.org/10.1016/j.foodhyd.2020.105718

[8] Guzzo, T., Mandaliti, W., Nepravishta, R., Aramini, A., Bodo, E., Daidone, I., Paci, M., et al. (2016) Conformational Change in the Mechanism of Inclusion of Ketoprofen in $\beta$-Cyclodextrin: NMR Spectroscopy, ab Initio Calculations, Molecular Dynamics Simulations, and Photoreactivity. The Journal of Physical Chemistry $B$, 120, 10668-10678. https://doi.org/10.1021/acs.jpcb.6b07913

[9] Yang, X., Zhao, Y., Chen, Y., Liao, X., Gao, C., Xiao, D., Yang, B., et al. (2013) Host-Guest Inclusion System of Mangiferin with $\beta$-Cyclodextrin and Its Derivatives. Materials Science and Engineering: C, 33, 2386-2391.

https://doi.org/10.1016/j.msec.2013.02.002

[10] Pereva, S., Nikolova, V., Sarafska, T., Angelova, S., Spassov, T. and Dudev, T. (2020) Inclusion Complexes of Ibuprofen and $\beta$-Cyclodextrin: Supramolecular Structure and Stability. Journal of Molecular Structure, 1205, Article ID: 127575. https://doi.org/10.1016/j.molstruc.2019.127575

[11] Ciulu-Costinescu, F., Podgoreanu, P., Delcaru, C., Simionescu, A., Georgescu, E.F., Bostan, M. and Chifiriuc, M.C. (2019) Antimicrobial Assay of a Capsaicin- $\alpha$-Cyclo- 
dextrin Inclusion Complex against Planktonic and Adherent Cells. Farmacia, 67, 496-503. https://doi.org/10.31925/farmacia.2019.3.18

[12] Guan, M.Y., Shi, R., Zheng, Y.Y., Zeng, X., Fan, W.Y., Wang, Y.G. and Su, W.W. (2020) Characterization, in Vitro and in Vivo Evaluation of Naringenin-Hydroxypropyl- $\beta$-Cyclodextrin Inclusion for Pulmonary Delivery. Molecules, 25, Article ID: 554. https://doi.org/10.3390/molecules25030554

[13] Ivansyah, A.L., Nurhidayah, E.S., Sundari, C.D.D., Martoprawiro, M.A. and Buchari, B. (2019) Computational Study of Inclusion Complex between Omeprazole Enantiomer and $\beta$-Cyclodextrin: NBO and RDG Analysis. Journal of Physics: Conference Series, 1402, Article ID: 055068. https://doi.org/10.1088/1742-6596/1402/5/055068

[14] Carneiro, S.B., Duarte, C., Ílary, F., Heimfarth, L., Quintans, S., de Souza, J., et al. (2019) Cyclodextrin-Drug Inclusion Complexes: In Vivo and in Vitro Approaches. International Journal of Molecular Sciences, 20, Article ID: 642. https://doi.org/10.3390/ijms20030642

[15] Gentili, A. (2020) Cyclodextrin-Based Sorbents for Solid Phase Extraction. Journal of Chromatography A, 1609, Article ID: 460654.

https://doi.org/10.1016/j.chroma.2019.460654

[16] García-Pérez, P., Losada-Barreiro, S., Gallego, P. P. and Bravo-Díaz, C. (2019) Cyclodextrin-Elicited Bryophyllum Suspension Cultured Cells: Enhancement of the Production of Bioactive Compounds. International Journal of Molecular Sciences, 20, Article ID: 5180. https://doi.org/10.3390/ijms20205180

[17] Paczkowska, M., Szymanowska-Powałowska, D., Mizera, M., Siąkowska, D., Błaszczak, W., Piotrowska-Kempisty, H. and Cielecka-Piontek, J. (2019) Cyclodextrins as Multifunctional Excipients: Influence of Inclusion into $\beta$-Cyclodextrin on Physicochemical and Biological Properties of Tebipenem Pivoxil. PloS ONE, 14, e0210694. https://doi.org/10.1371/journal.pone.0210694

[18] de Almeida Magalhães, T.S.S., de Oliveira Macedo, P.C., Kawashima Pacheco, S.Y., Silva, S.S.D., Barbosa, E.G., Pereira, R.R., et al. (2020) Development and Evaluation of Antimicrobial and Modulatory Activity of Inclusion Complex of Euterpe oleracea Mart Oil and $\beta$-Cyclodextrin or HP- $\beta$-Cyclodextrin. International Journal of Molecular Sciences, 21, Article ID: 942. https://doi.org/10.3390/ijms21030942

[19] da CunhaTrajano, V.C., Brasileiro, C.B., de Souza Henriques, J.A., de Miranda Cota, L., Lanza, C.R. and Cortés, M.E. (2019) Doxycycline Encapsulated in $\beta$-Cyclodextrin for Periodontitis: A Clinical Trial. Brazilian Oral Research, 33, Article ID: 0112. https://doi.org/10.1590/1807-3107bor-2019.vol33.0112

[20] Santos, C.I., Ribeiro, A.C. and Esteso, M.A. (2019) Drug Delivery Systems: Study of Inclusion Complex Formation between Methylxanthines and Cyclodextrins and Their Thermodynamic and Transport Properties. Biomolecules, 9, Article ID: 196. https://doi.org/10.3390/biom9050196

[21] Zhang, J.Q., Wu, D., Jiang, K.M., Zhang, D., Zheng, X., Wan, C.P., Lin, J., et al. (2015) Preparation, Spectroscopy and Molecular Modelling Studies of the Inclusion Complex of Cordycepin with Cyclodextrins. Carbohydrate Research, 406, 55-64. https://doi.org/10.1016/j.carres.2015.01.005

[22] Ren, Z., Xu, Y., Lu, Z., Wang, Z., Chen, C., Guo, Y., Zheng, Y., et al. (2019) Construction of a Water-Soluble and Photostable Rubropunctatin/ $\beta$-Cyclodextrin Drug Carrier. RSC Advances, 9, 11396-11405. https://doi.org/10.1039/C9RA00379G

[23] Ko, N.R., Van, S.Y., Hong, S.H., Kim, S.Y., Kim, M., Lee, J.S., Oh, S.J., et al. (2020) Dual pH-and GSH-Responsive Degradable PEGylated Graphene Quantum Dot-Based Nanoparticles for Enhanced HER2-Positive Breast Cancer Therapy. Na- 
nomaterials, 10, Article ID: 91. https://doi.org/10.3390/nano10010091

[24] Bezamat, J.M., Yokaichiya, F., Franco, M.K.D., Castro, S.R., de Paula, E. and Cabeça, L.F. (2020) Complexation of the Local Anesthetic Pramoxine with Hydroxypropyl-Beta-Cyclodextrin Can Improve Its Bioavailability. Journal of Drug Delivery Science and Technology, 55, Article ID: 101475.

https://doi.org/10.1016/j.jddst.2019.101475

[25] Moradi, S., Barati, A., Tonelli, A.E. and Hamedi, H. (2020) Chitosan-Based Hydrogels Loading with Thyme Oil Cyclodextrin Inclusion Compounds: From Preparation to Characterization. European Polymer Journal, 122, Article ID: 109303. https://doi.org/10.1016/j.eurpolymj.2019.109303

[26] Andriotis, E.G., Eleftheriadis, G.K., Karavasili, C. and Fatouros, D.G. (2020) Development of Bio-Active Patches Based on Pectin for the Treatment of Ulcers and Wounds Using 3D-Bioprinting Technology. Pharmaceutics, 12, Article ID: 56. https://doi.org/10.3390/pharmaceutics12010056

[27] Fenyvesi, E., Vikmon, M. and Szente, L. (2016). Cyclodextrins in Food Technology and Human Nutrition: Benefits and Limitations. Critical Reviews in Food Science and Nutrition, 56, 1981-2004. https://doi.org/10.1080/10408398.2013.809513

[28] Pourreza, N. and Naghdi, T. (2017) D-Limonene as a Green Bio-Solvent for Dispersive Liquid-Liquid Microextraction of $\beta$-Cyclodextrin Followed by Spectrophotometric Determination. Journal of Industrial and Engineering Chemistry, 51, 71-76. https://doi.org/10.1016/j.jiec.2017.02.016

[29] da Silva Júnior, W.F., de Oliveira Pinheiro, J.G., Moreira, C.D., de Souza, F.J. and de Lima, Á.A. (2017) Alternative Technologies to Improve Solubility and Stability of Poorly Water-Soluble Drugs. In: Grumezescu, A.M., Ed., Multifunctional Systems for Combined Delivery, Biosensing and Diagnostics, Elsevier, Singapore, 281-305. https://doi.org/10.1016/B978-0-323-52725-5.00015-0

[30] Banerjee, R., Sinha, R. and Purkayastha, P. (2019) $\beta$-Cyclodextrin Encapsulated Coumarin 6 on Graphene Oxide Nanosheets: Impact on Ground-State Electron Transfer and Excited-State Energy Transfer. ACS Omega, 4, 16153-16158. https://doi.org/10.1021/acsomega.9b02335

[31] Mie, G. (1908) Articles on the Optical Characteristics of Turbid Tubes, Especially Colloidal Metal Solutions. Annalen der Physik, 330, 377-445. https://doi.org/10.1002/andp.19083300302

[32] Gao, X.Y., Chen, G. and Ning, L.H. (2013) Plasmonic Characteristics of Nanorod-Based Metallic Nanostructures. Optics \& Laser Technology, 48, 394-400. https://doi.org/10.1016/j.optlastec.2012.10.036

[33] Mulvaney, P. (1996) Surface Plasmon Spectroscopy of Nanosized Metal Particles. Langmuir, 12, 788-800. https://doi.org/10.1021/la9502711

[34] Stephan, L. and El-Sayed, M.A. (1999) Spectral Properties and Relaxation Dynamics of Surface Plasmon Electronic Oscillations in Gold and Silver Nanodots and Nanorods. The Journal of Physical Chemistry B, 103, 8410-8426.

https://doi.org/10.1021/jp9917648

[35] Ralph, W. (2001) A Clearer Vision for in Vivo Imaging. Nature Biotechnology, 19, 316-317. https://doi.org/10.1038/86684

[36] Jiang, Z.M., Li, G.Y. and Zhang, M.X. (2017) A Novel Electrochemical Sensor Based on SH- $\beta$-Cyclodextrin Functionalized Gold Nanoparticles/Reduced-Graphene Oxide Nanohybrids for Ultrasensitive Electrochemical Sensing of Acetaminophen and Ofloxacin. International Journal of Electrochemical Science, 12, 5157-5173. https://doi.org/10.20964/2017.06.28 
[37] Shin, M.J. and Shin, J.S. (2020) A Molecularly Imprinted Polymer Undergoing a Color Change Depending on the Concentration of Bisphenol A. Microchimica Acta, 187, Article No. 44. https://doi.org/10.1007/s00604-019-4050-0

[38] Yang, Y. (2016) Electrochemical Sensor for Ultrasensitive Determination of Bisphenol ABased on Gold Nanoparticles/ $\beta$-Cyclodextrin Functionalized Reduced Graphene Oxide Nanocomposite. International Journal of Electrochemical Science, 11, 2778-2789. https://doi.org/10.20964/110402778

[39] Luo, S.X., Wu, Y.H., Mou, Q.S., Li, J.H. and Luo, X.X. (2019) A Thio- $\beta$-Cyclodextrin Functionalized Graphene/Gold Nanoparticle Electrochemical Sensor: A Study of the Size Effect of the Gold Nanoparticles and the Determination of Tetrabromobisphenol A. RSC Advances, 9, 17897-17904. https://doi.org/10.1039/C9RA02614B

[40] Manickam, P., Vashist, A., Madhu, S., Sadasivam, M., Sakthivel, A., Kaushik, A. and Nair, M. (2020) Gold Nanocubes Embedded Biocompatible Hybrid Hydrogels for Electrochemical Detection of $\mathrm{H}_{2} \mathrm{O}_{2}$. Bioelectrochemistry, 131, Article ID: 107373. https://doi.org/10.1016/j.bioelechem.2019.107373

[41] Yadav, M., Das, M., Bhatt, S., Shah, P., Jadeja, R. and Thakore, S. (2021) Rapid Selective Optical Detection of Sulfur Containing Agrochemicals and Amino Acid by Functionalized Cyclodextrin Polymer Derived Gold Nanoprobes. Microchemical Journal, 169, Article ID: 106630. https://doi.org/10.1016/j.microc.2021.106630

[42] Neri, G., Cordaro, A., Scala, A., Cordaro, M., Mazzaglia, A. and Piperno, A. (2021) PEGylated Bis-Adamantane Carboxamide as Guest Bridge for Graphene Poly-Cyclodextrin Gold Nanoassemblies. Journal of Molecular Structure, 1240, Article ID: 130519. https://doi.org/10.1016/j.molstruc.2021.130519

[43] Kapan, B., Kurbanoglu, S., Esenturk, E.N., Soylemez, S. and Toppare, L. (2021) Electrochemical Catechol Biosensor Based on $\beta$-Cyclodextrin Capped Gold Nanoparticles and Inhibition Effect of Ibuprofen. Process Biochemistry, 108, 80-89. https://doi.org/10.1016/j.procbio.2021.06.004

[44] Wu, H., Fang, F., Wang, C., Hong, X., Chen, D. and Huang, X. (2021) Selective Molecular Recognition of LowDensity Lipoprotein Based on $\beta$-Cyclodextrin Coated Electrochemical Biosensor. Biosensors, 11, Article ID: 216. https://doi.org/10.3390/bios11070216

[45] Olmo, J.A.D., Ruiz-Rubio, L., Pérez-Alvarez, L., Sáez-Martínez, V. and Vilas-Vilela, J.L. (2020) Antibacterial Coatings for Improving the Performance of Biomaterials. Coatings, 10, Article ID: 139. https://doi.org/10.3390/coatings10020139

[46] Raquel, B., Nerín, C. and Filomena, S. (2020) Encapsulation Systems for Antimicrobial Food Packaging Components: An Update. Molecules, 25, Article ID: 1134. https://doi.org/10.3390/molecules25051134

[47] Bindhu, M.R., Saranya, P., Sheeba, M., Vijilvani, C., Rejiniemon, T.S., Al-Mohaimeed, A.M., AbdelGawwad, M.R. and Elshikh, M.S. (2021) Functionalization of Gold Nanoparticles by $\beta$-Cyclodextrin as a Probe for the Detection of Heavy Metals in Water and Photocatalytic Degradation of Textile Dye. Environmental Research, 201, Article ID: 111628. https://doi.org/10.1016/j.envres.2021.111628

[48] Qu, H.N., Yang, L.R., Yu, J.M., Dong, T.T., Rong, M., Zhang, J.F., Xing, H.F., Wang, L., Pan, F. and Liu, H.Z. (2017) A Redox Responsive Controlled Release System Using Mesoporous Silica Nanoparticles Capped with Au Nanoparticles. RSC Advances, 7, 35704-35710. https://doi.org/10.1039/C7RA04444E

[49] Trapani, M., Scala, A., Mineo, P.G., Pistone, A., Díaz-Moscoso, A., Fragoso, A., Scolaro, L.M. and Mazzaglia, A. (2021) Thiolated Amphiphilic $\beta$-Cyclodextrin-Decorated Gold Colloids: Synthesis, Supramolecular Nanoassemblies and Controlled 
Release of Dopamine. Journal of Molecular Liquids, 336, Article ID: 116880. https://doi.org/10.1016/j.molliq.2021.116880

[50] Qiu, J.R., Kong, L.D., Cao, X.Y., Li, A.J., Wei, P., Lu, W., Mignani, S., Caminade, A.M., Majoral, J.P. and Shi, X.Y. (2018) Enhanced Delivery of Therapeutic siRNA into Glioblastoma Cells Using Dendrimer-Entrapped Gold Nanoparticles Conjugated with $\beta$-Cyclodextrin. Nanomaterials, 8, Article ID: 131. https://doi.org/10.3390/nano8030131

[51] Pestovsky, Y.S. and Martínez-Antonio, A. (2018) Gold Nanoparticles with Immobilized $\beta$-Cyclodextrin-Capsaicin Inclusion Complex for Prolonged Capsaicin Release. IOP Conference Series: Materials Science and Engineering, 389, Article ID: 012030. https://doi.org/10.1088/1757-899X/389/1/012030 